TITLE:

\title{
Single-valued Wave Function of Aharonov-Bohm Problem
}

$\operatorname{AUTHOR}(\mathrm{S})$ :

Kawamoto, Hiroshi

\section{CITATION:}

Kawamoto, Hiroshi. Single-valued Wave Function of Aharonov-Bohm Problem. 数理解析 研究所講究録 1997, 980: 162-170

ISSUE DATE:

1997-02

URL:

http://hdl.handle.net/2433/60862

RIGHT: 
Single-valued Wave Function of Aharonov-Bohm Problem

$$
\begin{aligned}
& \text { Hiroshi Kawamoto } \\
& \text { (川本宏) }
\end{aligned}
$$

Maritime Safety Academy, Transportation Ministry of Japan,

Kure, Hiroshima 737

(Abstract)

The wave function of a charged particle orbiting around a confined magnetic flux is single-valued. On the basis of this, the Aharonov-Bohm effect is studied - In conclusion, it is found that the flux-dependent diffraction originated from the interference between the two splitted waves is impossible although the amplitude of each wave function is a periodic function of the applied flux. Futhermore, we obtain a greatly depressed cross section for a rigid wall tube. (Text)

There has been a controversy whether the wave function of a particle orbiting around a confined magnetic flux $\Phi$ is single-valued ${ }^{1) .2)}$ or multi-valued. ${ }^{3) . ~ 4)}$ In this paper, the single-valued wave function is obtained. On the basis of a single-valued wave function, we examine the so-called Aharonov-Bohm effect ${ }^{5)}$, consisting of three issues; (i) a charged beam splitted into two parts gives rise to a $\Phi$-dependent diffraction pattern ${ }^{3)}$ 1). 5), (ii) an energy eigenvalue of a particle is influenced by $\Phi^{61,7), 8)}$, (iii) a scattering cross section of the beam is a function of $\Phi^{51,9), 10)}$.

Our wave function $\Psi(\vec{r}, t)$ satisfies the Schrödinger equation $i \hbar \partial \Psi /$ $\partial t=H \Psi$, where the Hamiltonian $H$ is

$$
H=\frac{1}{2 \mu}(-i \hbar \nabla-q \vec{A})^{2}+q \phi
$$

In the present analysis, the magnetic flux $\Phi$ is confined within a rigid tube of radius $a$ running along $z$-axis. Therefore the wave function $\Psi(\vec{r}, t)$ is defined in a region $r \geqq a$. Let us divide $\vec{A}(\vec{r}, t)$ into two parts, $\vec{A}(\vec{r}, t)=$ 
$\overrightarrow{A_{0}}(\vec{r})+\overrightarrow{A_{1}}(\vec{r}, t)$, where $\vec{A}_{0}$ and $\overrightarrow{A_{1}}$ respectively represent a d.c part and an a.c part of the total $\vec{A}$. Under the condition $\nabla \times \vec{A}=0$, the wave function takes a factorized form

$$
\Psi(\vec{r}, t)=\exp \left[i q \int_{\vec{r}_{0}}^{\vec{r}} \vec{A}\left(\vec{r}^{\prime}, t\right) d \vec{r}, / \hbar\right] \psi(\vec{r}, t) .
$$

The pseudo wave function $\phi(\vec{r}, t)$ satisfies a pseudo Schrödinger equation

$$
i \hbar \frac{\partial}{\partial t} \psi(\vec{r}, t)=\left[-\frac{\hbar^{2}}{2 \mu} \nabla^{2}-q \int_{\vec{r}_{0}}^{\vec{r}} \vec{E}_{1}\left(\vec{r}^{\prime}, t\right) d \vec{r},\right] \psi(\vec{r}, t),(3)
$$

where $\phi(\vec{r}, t) \equiv 0$ has been chosen. To obtain Eq. ( 3$)$, a path-independent equality $\nabla \int_{\vec{r}_{0}}^{\vec{r}} \vec{A} d \vec{r},=\vec{A}(\vec{r}, t)$ yielded from $\nabla \times \vec{A}=0$ has been used.

With the use of Faraday's law $\oint \vec{E}_{1} d \vec{r}=-\partial \Phi_{1}(t) / \partial t$ in Eq. ( 3 ), we obtain a multi-valuedness of $\phi(\vec{r}, t)$,

$$
\phi(r, \theta+2 \pi, t)=\exp \left[-2 \pi i \alpha_{1}(t)+i \lambda\right] \phi(r, \theta, t) .(4)
$$

Here $\alpha_{1}(t)$ is a scaled flux $\alpha_{1}(t) \equiv q \Phi_{1}(t) / h^{\prime}$, and $\lambda$ is an integral constant composed of two parts, $\lambda=\lambda_{0}+\lambda^{\prime}$. A part $\lambda_{0}$ is originated from $\vec{A}_{0}(\vec{r})$ and plays an essential role of the problem, while $\lambda$ ' is an irrelevant part. In this paper, we set $\lambda^{\prime}=0$. For the purpose of finding the explicit form of $\lambda_{0}$, let us consider a case where only the static vector potential $\overrightarrow{A_{0}}(\vec{r})$ exists. In this case, the pseudo Schrödinger equation ( 3 ) takes the form of free particle's type. However $\phi(\vec{r}, t)$ is governed by the (static) magnetic flux $\Phi_{0}$. This can be shown if the system is rotated on the axis of the flux tube. In the system rotating at an angular velocity $2 \pi / T$, our $\vec{r}_{0}$ in the factorized phase also rotates with the same angular velocity. Thus we obtain the pseudo Schrodinger equation $i \hbar \frac{\partial}{\partial t} \psi_{r}(\vec{r}, t)=\left[-\frac{\hbar^{2}}{2 \mu} \nabla^{2}-q \frac{\partial}{\partial t} \vec{r}_{0}(t) \cdot \vec{A}_{0}\left(\vec{r}_{0}(t)\right)\right] \psi_{r}(\vec{r}, t)$. 
The solution of Eq. ( 5$)$ has a form $\psi_{r}(\vec{r}, t)=\exp (i \gamma(t)) \chi_{0}(\vec{r}, t)$ where $r(t)$ represents the rotation of the system

$$
\hbar \frac{\partial}{\partial t} \gamma(t)=a \vec{A}_{0}\left(\vec{r}_{0}(t)\right) \cdot \frac{\partial}{\partial t} \vec{r}_{0}(t)
$$

while $\chi_{0}(\vec{r}, t)$ is a solution of the free Schrödinger equation $i \hbar \partial \chi_{0} / \partial$ $t=-\hbar^{2} / 2 \mu \cdot \nabla^{2} \chi_{0}$ in the rest frame.

Integrating Eq. (6) along a path $\vec{r}_{0}(t)$ going around the flux tube, we have $\Delta \gamma \equiv \gamma(T)-\gamma(0)=-2 \pi \alpha_{0}$. A sign of the equality reflects the direction of the path $\vec{r}_{0}(t)$ which is opposite with respect to the direction $\theta \rightarrow \theta+2 \pi$ introduced in Eq. (4). Thus the pseudo wave function $\phi_{r}(\vec{r}, t)$ acquires an additional phase $\Delta \gamma=-2 \pi \alpha_{0}$ after a revolution of $\vec{r}_{0}(t)$;

$$
\phi_{r}(r, \theta, t+T)=\exp \left(-2 \pi i \alpha_{0}\right) \psi_{r}(r, \theta, t) .(7)
$$

The additional phase $\Delta y=-2 \pi \alpha_{0}$ in Eq. ( 7 ) could be interpreted, in a wide sense, as Berry's phase ${ }^{\prime \prime}$. The reason why the opposite direction of $\vec{r}_{0}$ $(t)$ is chosen is that the rotation of the system in such a direction, $\theta_{0}(t$ )$\rightarrow \theta_{0}(t+T)=\theta_{0}(t)-2 \pi$, is physically equivalent to the coordinate transformation $\theta \rightarrow \theta+2 \pi$. In other words, the role of $\theta \rightarrow \theta+2 \pi$ can be replaced by $\theta_{0}(t) \rightarrow \theta_{0}(t+T)=\theta_{0}(t)-2 \pi$ ! The validity of this replacement is readily seen from the explicit form of the factorized phase in Eq $.(2), \int_{\theta_{0}(t)}^{\theta} A_{0}\left(r^{\prime}\right) r^{\prime} d \theta^{\prime}=\Phi_{0} / 2 \pi \cdot\left(\theta-\theta_{0}(t)\right)$ obtained from $\nabla \times$ $\vec{A}=0$. Considering this equivalence we have, from $E q .(7), \lambda_{0}=-2 \pi \alpha_{0}$ in Eq. ( 4 ). As to such an equivalence, a more general viewpoint is possible. That is, the present equivalence is merely an example of the equivalence between the rotation of a system and a transformation $\theta \rightarrow \theta+2 \pi$, which is widely accepted in the theory of quantum mechanics ${ }^{12}$. Hence, we are led to the conclusion that the wave function $\Psi(\vec{r}, t)$ given in Eq. (2) is single-valued. That is, an 
additional phase $2 \pi a(t)$ of the factorized phase factor for the revolution $\theta_{0}(t) \rightarrow \theta_{0}(t)-2 \pi$ (or equivalently $\theta \rightarrow \theta+2 \pi$ ), is exactly cancelled out by the acquired phase $-2 \pi \alpha_{1}(t)+\lambda_{0}\left(\lambda_{0}=-2 \pi \alpha_{0}\right)$ of the pseudo wave function $\phi(\vec{r}, t)$.

The single-valuedness of $\Psi(\vec{r}, t)$ ( for a static $\overrightarrow{A_{0}}(\vec{r})$ ) is found by another method. Let us consider a particle orbiting around the flux $\Phi$ within a loop of radius $\xi_{0}$. Here the flux $\Phi$ is assumed to switch over from an a.c $\Phi_{1}(t)$ to a d.c $\Phi_{0}$ at $t=0$. Fortunately, the exact solution of Eq. ( 3 ) for $t<0$ is obtained ;

$$
\phi_{\mathrm{n}}(\theta, t)=N \exp \left[-i \int_{t_{0}}^{t} E_{\mathrm{n}}\left(t^{\prime}\right) d t^{\prime} / \hbar\right] \beta_{\mathrm{n}}(\theta, t) .
$$

where $\beta_{n}(\theta, t)=\exp \left[i\left(n-\alpha_{1}(t)\right) \theta\right]$ and $E_{n}(t)=\hbar^{2}(n-$ $\left.a_{1}(t)\right)^{2} / 2 \mu \xi_{0}{ }^{2}$. It is important to note that $\phi_{\text {a }}$ satisfies the circular condition (4) with $\lambda_{0}=0$. Since there is no d.c part of $\vec{A}(\vec{r}, t)$ in $t<0, \lambda_{0}=0$ of $\phi_{\mathrm{n}}$ is reasonable. Thus the wave function given by $E q$. ( 2 ) is single-valued. The solution ( 8 ) develops into, as $\Phi_{1}(t)$ smoothly approaches to $\Phi_{0}$, an extrapolated form $\phi_{\mathrm{a} o}(\theta, t)=N \exp \left[-i E_{\mathrm{n}}(0) t / \hbar\right.$ ] $\exp \left[i\left(n-\alpha_{0}\right) \theta\right]$. This extrapolated form ( of the pseudo wave function ) is consistent with the wave function really existing in the region $t \geqq 0$. The eigenvalue equation in $t \geqq 0$ is

$$
-\frac{\hbar^{2}}{2 \mu \xi_{0}^{2}}\left(\frac{\partial}{\partial \theta}-i \alpha_{0}\right)^{2} \theta_{n i}(\theta)=E_{n i} \theta_{n i}(\theta)
$$

Equation ( 9 ) has, formally, many solutions $\theta_{n j}(\theta)=\exp \left(i l_{n j} \theta / \hbar\right)$ where $l_{n j}$ are canonical angular momenta $l_{n j}=\left(n-j \alpha_{0}\right) \hbar(n$ and $j$ are 0 , $\pm 1, \pm 2, \pm 3, \cdots)$. The energy eigenvalues of $\theta_{n j}$ are $E_{n j}=\left(l_{n j}-\right.$ $\left.\hbar \alpha_{0}\right)^{2} / 2 \mu \xi \sigma_{0}^{2}$. Among these solutions, there is one solution physically realistic. To find this, let us consider the conservation law of energy-angular momentum, which is satisfied at the linking of two wave functions respectively 
given in the regions $t<0$ and $t \geqq 0$. Under this restriction, the wave function in $t<0$. which is single-valued, links up only to $\theta_{n o}(\theta)$ also singlevalued. Therefore we are led to the single-valued wave function.

Let us examine $(i) \sim($ in $)$. (i) An incident wave is splitted into two waves $\Psi_{c_{1}}$ and $\Psi_{c_{2}}$ travelling along the right-hand $\left(C_{1}\right)$ and the left-hand $\mathrm{C}_{2}$ ) of the flux. These splitted waves get together at a point $P$ on a screen, $\Psi_{c_{1}+c_{2}}(P)=\Psi_{c_{1}}(P)+\Psi_{c_{2}}(P)$. According to the existing theory where the pseudo wave function is assumed to be single-valued, $\left|\Psi_{c:+c .2}(P)\right|^{2}=4$ $|Q(P)|{ }^{2} \cos ^{2}\left(\pi \alpha_{0}\right){ }^{31.4)}$. 5) is obtained. The factor $\cos ^{2}\left(\pi \alpha_{0}\right)$ comes from the interference phases $\pm q \oint_{c_{1-c 2}} \vec{A}_{0} d \vec{r}, / \hbar= \pm 2 \pi \alpha_{0}$ appearing in the mixed terms $\Psi_{c_{1}}(P) \Psi^{*} c_{c}(P)$ and $\Psi^{*} c_{1}(P) \Psi_{c_{2}}(P)$. Contrary to this. such an interference is cancelled out, in the present theory, by the counter-interference phases $\mp 2 \pi \alpha_{0}$ respectively arising from $\phi_{\mathrm{cl}}($ P) $\phi_{c_{2}} *(P)$ and $\phi^{*} c_{1}(P) \psi_{c_{2}}(P)$. Hence we have $\left|\Psi_{c_{1}+c_{2}}(P)\right|^{2}=$ $4|Q(P)|^{2}$. The present $Q(P)$ is expressed by $Q(P)=\sum_{n=-\infty}^{\infty} \exp \left(\right.$ in $\left.\theta_{p}\right)$ $\left[a_{n}(|\nu|) J_{-|\nu|}\left(r_{p}\right)+b_{n}(|\nu|) J_{|\nu|}\left(r_{p}\right)\right]$. Here the Bessel functions $J_{ \pm \text {iul }}(r)$ satisfy the radial part of the equation,

$$
-\frac{\hbar^{2}}{2 \mu}\left[\frac{\partial}{r \partial r}\left(r \frac{\partial}{\partial r}\right)-\frac{|\nu|^{2}}{r^{2}}\right] R(r)=E R(r), \quad\left(\nu \equiv n-\alpha_{0}\right) .(10)
$$

The $\boldsymbol{\alpha}_{0}$-dependence of the present $|Q(P)|^{i}$ is quite similar to the one of $\mid$ $\left.\Psi_{c_{1}+c_{2}}(P)\right|^{2}=4|Q(P)|^{2} \cos ^{2}\left(\pi \alpha_{0}\right)$, because it is, as readily seen, a periodic function of $\alpha_{0}$ with a period $\alpha_{0}=I$. Therefore, it seems important that the special care should be used, even in the excellent experiment 13), not to confuse the observed $\alpha_{0}$-dependence of $|Q(P)|^{2}$ with the ( unreal $) \alpha_{0}$-dependent interference expressed by $\cos ^{2}\left(\begin{array}{ll}\pi & a_{0}\end{array}\right)$.

(ii) It is found that the energy eigenvalue is a function of $\alpha_{0}$, because it is determined by Eq. ( 10$)$ which explicitly contains $\alpha_{n}$. An example has been already given in Eq. ( 9 ). Here, let us give a brief comment on the equality $j=$ - $q \partial E(\Phi) / \partial \Phi{ }^{6} \cdot 11$. A probability current density $\vec{j}(\vec{r}, t)$ 
satisfying $\partial \rho / \partial t+\nabla \cdot \vec{j}=0\left(\rho=|\Psi|^{2}\right.$ and $\vec{j}=(\hbar / 2 \mu i)\left[\Psi^{*} \nabla \Psi-\right.$ $\left.\left.\Psi \nabla \Psi^{*}\right]-(q / \mu)|\Psi|^{2} \vec{A}\right)$ is

$$
\vec{j}(\vec{r}, t)=\frac{\hbar}{2 \mu i}\left[\phi^{*}(\vec{r}, t) \nabla \phi(\vec{r}, t)-\phi(\vec{r}, t) \nabla \phi^{*}(\vec{r}, t)\right] .(11)
$$

If a particle confined within a loop of radius $\xi_{0}$ is considered, we have, from Eq. ( 11$), j=\hbar\left(n-\alpha_{0}\right) / 2 \pi \mu \xi_{0}{ }^{2}$ for a normalized pseudo wave function $|\phi|^{2}=\left(2 \pi \xi_{0}\right)^{-1}$. Combining this with $E_{\mathrm{n}}=\hbar^{2}\left(n-\alpha_{0}\right)^{2} / 2 \mu \xi_{0}{ }^{2}$ . we reach $j=-q \partial E(\Phi) / \partial \Phi$. An angular momentum $l=\int_{0}^{2 \pi}\left(\xi_{0} \times \mu j\right) \xi_{0}$. $d \theta=\hbar\left(' n-\alpha_{0}\right)$ yielded from $\vec{j}(\vec{r}, t)$ is a kinematical one 14). Contrary to this, the canonical angular momentum is $l_{\text {can }}=\hbar n^{2)}$, as seen from $l_{\mathrm{kin}}=$ $\hbar\left(n-a_{0}\right)$.

(iii) Supposing that it seems considerably difficult to measure the cross section in the actual condition, let us study, providing for the future, the differential cross section $\sigma(\theta)$. For the thin filament $(a=0), \sigma(\theta)$ $=\sin ^{2}\left(\pi \alpha_{0}\right) / \pi k \sin ^{2}(\theta / 2)^{5), 9), 10)}$ has already been given. In the present case where the radius of flux tube is finite, $\sigma(\theta)$ is depressed by the additional $k$-dependence, while the $\alpha_{0}$-dependence is basically the same. Since the problem can be studied either by the wave function or by the pseudo wave function, the pseudo wave function will be used. An incident particle moving in a $x$-direction $u ;(r, \theta)=\exp (i k r \cos \theta)$ is represented by

$$
u_{i}(r, \theta)=\sum_{n=-\infty}^{\infty} \exp (i n \pi / 2) J_{n}(k r) \exp (i n \theta) \text {, }
$$

which carries a current density $j=\hbar k / \mu$ ( see Eq. $(I 1))$. On the other hand, the scattered wave is represented by

$$
u_{s}(r, \theta)=\sum_{n=-\infty}^{\infty} A_{n}(\nu) \exp (i n \pi / 2) H_{|\nu|}^{(1)}(k r) \exp (i \nu \theta),(13)
$$

where $H_{|\nu|}{ }^{(1)}(k r)$ describes the outgoing wave and $\exp (i \nu \theta)$ reflects the 
multi-valuedness of the pseudo wave function. From the inpenetrating condition of the flux tube, $u_{i}(a, \theta)+u_{s}(a, \theta)=0$, we obtain $A_{n}(\nu)=$ $A_{n}{ }^{\prime}(\nu) \exp (-i n \pi / 2)$;

$A_{n}^{\prime}(\nu)=\frac{-i \sin (\pi \nu) \sin (\pi|\nu|)}{\pi\left[J_{-|\nu|}(k a)-e^{-i \pi|\nu|} J_{|\nu|}(k a)\right]} \sum_{m=-\infty}^{\infty} \frac{(-i)^{m} J_{m}(k a)}{m-\nu} \cdot(14)$

In Eq. ( 14$)$ we have used $\sin (\pi|\nu|) \cdot H_{|\nu|}{ }^{(1)}(z)=-i[J-|\nu|(z)-$ $\left.\exp (-i \pi|\nu|) J_{|\nu|}(z)\right]$. Let us note $A_{n}^{\prime}(\nu)=A_{n}^{\prime}(|\nu|)$ resulting from $J_{-m}(z)=(-1)^{m} J_{m}(z)$.

Considering the asymptotic behavior of $H_{|\nu|}{ }^{(1)}(z)$ at $z \rightarrow \infty, H_{|\nu|}{ }^{(1)}(z)$ $\rightarrow(2 / \pi z)^{1 / 2} \exp [i(z-(2|\nu|+1) \pi / 4)]$, we have a scattering amplitude $f(\theta)$, defined by $u_{s}(r, \theta) \rightarrow \exp (i k r) f(\theta) / \sqrt{r}(r \rightarrow \infty$ ) , as $f(\theta)=(2 / \pi k)^{1 / 2} \exp (-i \pi / 4) \cdot \Sigma A_{n}^{\prime}(\nu) \exp (-i|\nu|$ $\pi / 2] \cdot \exp (i \nu \theta)$. Thus an expression of the differential cross section $\sigma(\theta) \equiv|f(\theta)|^{2}$ is

$\sigma(\theta)=\frac{2}{\pi k} \cdot\left|\sum_{n=-\infty}^{\infty} A_{n}^{\prime}(|\nu|) \exp (-i \pi|\nu| / 2) \exp (i \nu \theta)\right|^{2} \cdot(15)$

It is found that $\sigma(\theta)$ is a periodic function of $a_{0}$ ( $\alpha_{0}$ will be restricted within $0<\alpha_{0}<1$ ). If some linear combination of ( positive order) $J_{|\nu|}($ $k r)$ is adopted in place of $H_{|\nu|}^{(1)}(k r)$, the expression for $a=0$ 5).9). (10) is reproduced from Eq. ( 15 ).

Let us study the interesting case $k a \ll l$ where $J_{0}(k a) \leftrightharpoons l_{\text {and }} J_{\mathrm{n}}$ ( $k a) \leftrightharpoons O(n \neq 0)$. In this case, an expression of $\sigma(\theta)$, because of $|\nu|$ $\nu \cdot \sin (\pi \nu)=\sin (\pi|\nu|)$, becomes

$\sigma(\theta)=2 / \pi k \cdot\left|\sum_{n=-\infty}^{\infty} \sin (\pi|\nu|) \exp (i \nu \theta) / D(k ;|\nu|)\right|^{2}(16)$

where 


$$
\begin{gathered}
D(k ;|\nu|)=(k a / 2)^{-|\nu|} \exp (i \pi|\nu| / 2) \Gamma(1+ \\
-(k a / 2)^{|\nu|} \exp (-i \pi|\nu| / 2) \Gamma(1-|\nu|)
\end{gathered}
$$

Here the equalities $\Gamma(\nu) \Gamma(1-\nu)=\pi / \sin (\pi \nu)(\nu \neq 0, \pm 1, \pm 2$, $\cdots \cdots)$ and $\nu \Gamma(\nu)=\Gamma(I+\nu)$ have been used to rewrite the expansion $J_{ \pm|\nu|}$

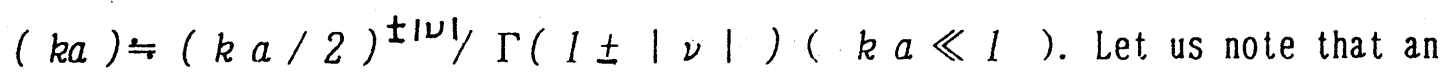
additional $k$-dependence, besides the factor $2 / \pi k$, appears in $\sigma(\theta)$ through the denominator $D(k ;|\nu|)$. This differs from $\sigma(\theta)$ obtained for the flux filament with $a=0$. Unfortunately, it seems formidably difficult to carry out the summation in Eq. $(16)$. Therefore we restrict our analysis to the $s$-component of $\sigma(\theta)$ whose contribution is principal in $k a \ll 1$. In a nonmagnetic region $\alpha_{0} \ll k a \ll 1$, we have

$$
\sigma_{0}=\frac{\pi}{2 k} \cdot|\gamma-i \pi / 2+\ln (k a / 2)|^{-2}\left[1+\left(\pi \alpha_{0} / 2\right)^{2}\right]^{-1}(18)
$$

where $\Gamma(1+z)=1-\gamma z+\cdots$ has been used $(\gamma$ is Euler's number $\gamma=$ $0.57721 \cdots \cdots)$. On the other hand, an interesting result is found in the magnetic region $k a \ll \alpha_{0}(k a \ll 1)$;

$$
\sigma_{0}=\frac{2}{\pi k} \cdot \sin ^{2}\left(\pi \alpha_{0}\right)\left[(k a / 2)^{\alpha_{0}} / \Gamma\left(1+\alpha_{0}\right)\right]^{2}
$$

Comparing this expression with $\sigma_{0}$ obtained from $\sigma(\theta)=\sin ^{2}\left(\pi \alpha_{0}\right) / \pi$ $k \sin ^{2}(\theta / 2)=2 \sin ^{2}\left(\pi \alpha_{0}\right) / \pi k \cdot\left[1+\cos \theta+(\cos \theta)^{2}+\cdots\right.$ -.. ${ }^{2}$, it is found that the present $\sigma_{0}$ is modified by the depressing factor $(k a / 2)^{2 \alpha_{0}}\left(\operatorname{note} \Gamma(1)=\Gamma(2)=1\right.$ and $\Gamma\left(1+\alpha_{0}\right) \leftrightharpoons 1$ for $0<\alpha_{0}$ $(1)$. The depression by $(k a / 2)^{2 \alpha_{0}}$ or by $|\ln (k a / 2)|^{-2}$ seems reasonable. The incident wave, as widely known to the physicists, becomes more insensitive to the tube as its wave length becomes longer. The depression in the magnetic region, explicitly depending on the value of $\alpha_{0}$, is much larger than 
the one arising in the case $\alpha_{0}=0$ ( see Eq. (18)). Hence such an extraordinarily small $\sigma_{0}$ can be considered as an experimental evidence of the $\Phi$ - dependent scattering, if it is observed in the magnetic region.

The author would like to thank Professor S. Takagi for helpful discussions. He is also grateful to colleagues of Kyoto University for stimulating discussions and the critical check of the calculations.

\section{References}

1) M. V. Berry, Eur. J. Phys. 1, 240 (1980)

2) R. Jackiw and A. N. Redlich, Phys. Rev. Lett. 50, 555 (1983).

3) M.P.Silverman, Phys. Rev. Lett. 51, 1927 (1983).

4) J.Q. Liang, Phys. Rev. Lett.53, 859 (1984).

5) Y. Aharonov and D. Bohm, Phys. Rev. 115, 485 (1959).

6) N. Byers and C.N. Yang, Phys. Rev. Lett. 7, 46 (1961).

7) R. B. Laughlin, Phys. Rev. B 23, 5632 (1981).

8) M. Büttiker, Y. Imry and R. Landauer, Phys. Lett. 96 A, 365 (1983).

9) T. Takabayashi, Suppl.Hadronic J.1, 219 (1985).

10) C. R. Hagen, Phys. Rev. Lett. 64, 503 (1990)

11) M. V. Berry, Proc. R. Soc. Lond. A 392, 45 (1984).

12) A. Messiah, Quantum Mechanics, Chap. 13, Amsterdam: North-Holland, 1959.

13) A. Tonomura, N. Osakabe, T. Ma tsuda, T. Kawasaki, J.Endo, S. Yano and H. Yamada, Phys. Rev. Lett. 56 (1986), 792.

14) F. Wilczek, Phys. Rev. Lett. 48, 1144 (1982). 